\title{
Esophagectomy without thoracotomy: 25 years of experience over 750 patients
}

\author{
Panagiotis Yannopoulos • Panagiotis Theodoridis • \\ Konstantinos Manes
}

Received: 21 November 2008 / Accepted: 16 March 2009/Published online: 7 April 2009

(C) The Author(s) 2009. This article is published with open access at Springerlink.com

\begin{abstract}
Objective This study aimed to review and evaluate our experience in 750 patients, who underwent transhiatal esophagectomy (THE) and analyze our data. Special attention was paid to some strategies, which we developed in the course of time, regarding the postoperative management of these patients and formulation of improved guidelines.

Patients and methods This is a retrospective analysis of all THE operations performed between January 1981 until May 2007 in 750 patients: 60 patients (8\%) had benign lesions, while $690(92 \%)$ had malignant ones $(5.2 \%$ of malignancies were located in the upper esophagus, $7.4 \%$ in the middle esophagus, $19 \%$ in the lower esophagus, and $68.4 \%$ at the cardioesophageal junction). THE and esophageal reconstruction were performed at the same operation in all patients. The stomach was our esophageal substitute of first choice with the colon and jejunum being acceptable alternatives in patients with prior gastric surgery and those necessitating synchronous gastrectomy for cancer invasion. A gastric tube was used as an esophageal substitute in 624 patients $(83.2 \%)$, the whole stomach in $70(9.4 \%)$, the colon in $43(5.73 \%)$, and a jejunal loop in $13(1.73 \%)$.

Results The overall in-hospital mortality rate was $2.93 \%$ (22 patients). There was no intraoperative death. Major compli-
\end{abstract}

P. Yannopoulos $\cdot$ P. Theodoridis $\cdot$ K. Manes

Esophageal Surgery Unit, “Athens Medical Center" Hospital,

5-7 Distomou str.,

15125 Athens, Greece

K. Manes $(\bowtie)$

31 Metamorphoseos str.,

15234 Athens, Greece

e-mail: dinos_manes@yahoo.com cations included atelectasis or pneumonia $(4.8 \%)$, pleural effusion $(22.7 \%)$, myocardial infarction $(0.5 \%)$, recurrent laryngeal nerve paralysis $(1.33 \%)$, and three tracheal lacerations $(0.4 \%)$. The anastomotic leak rate decreased gradually over time from $29.4 \%$ to $11.1 \%$ in the last 6 years. The average intraoperative blood loss was $315 \mathrm{ml}$ and $82 \%$ of the patients did not receive any blood transfusion. Late functional results were good or excellent in $93 \%$. The average length of hospital stay was 11 days and intensive care unit stay was 2.3 days. The actuarial 5 -year survival rate after THE for carcinoma was $21 \%$.

Conclusion THE is a safe and effective method of esophageal resection with low morbidity and mortality rates and good functional results when performed by experienced surgeons. We believe that our strategies concerning the way of dissecting the cervical esophagus, avoidance of performing pyloromyotomy, the delayed removal of the cervical drain and the delayed advance to oral feeding have reduced, noticeably, morbidity and mortality in our series.

Keywords Esophagus - Esophageal cancer .

Transhiatal esophagectomy (THE)

\section{Introduction}

Orringer and Sloan [1], in their article on transhiatal esophagectomy (THE) without thoracotomy dated in 1978, proposed this technique for the treatment of benign and malignant diseases of esophagus. Since then, numerous surgeons have adopted THE as an optional method of esophagectomy, as it offers several advantages over the transthoracic approach, namely avoidance of thoracotomy 
and the life-threatening complication of an anastomotic leak within the chest. [2-4].

In this study, we present and analyze our data with special attention to some strategies regarding the postoperative management of these patients.

\section{Patients and methods}

Between January 1, 1981 and May 31, 2007, a total of 750 patients underwent THE for benign and malignant diseases of the esophagus.

All these operations were performed by the same surgeon (Professor P. Yannopoulos).

Transhiatal esophagectomy was performed via a midline laparotomy and left cervical incision in accordance to Orringer and Sloan's [1] technique, as described in 1978, with a hand-sewn cervical anastomosis. Esophageal resection and reconstruction were performed at one sitting. The esophageal substitute of first choice was either a long gastric tube created from the greater curvature of the stomach or the whole stomach. We have used a gastric tube in 624 patients $(83.2 \%)$ and the whole stomach in 70 patients $(9.4 \%)$. Fortythree patients $(5.73 \%)$ required colonic pull-up due to prior gastric surgery or concomitant gastrectomy. A jejunal loop was used as an esophageal substitute in 13 patients $(1.73 \%)$. For patients with malignancy, the original esophageal bed in the posterior mediastinum was used for esophageal substitution in 635 patients $(92.03 \%)$. The substernal route was used in 49 patients $(7.10 \%)$, while the subcutaneous route was used in six patients $(0.87 \%)$.

Celiac axis and lower periesophageal lymph nodes were routinely removed during esophagectomies for malignancy. In the early years, our group used to add pyloromyotomy to the procedure, which was abandoned later. No chest tubes were inserted routinely. All patients were given a feeding jejunostomy.

The preoperative workup included a barium esophagogram, endoscopic examination, and biopsy for the malignant group and a full blood count, routine serum chemistry, chest radiograph, cardiologic examination, and respiratory function tests in smokers and patients over 70 years old. Additionally, chest and abdominal computed tomography with intravenous contrast medium was performed in the malignant group. Endoscopic ultrasonography in cases of highly suspicious $\mathrm{T} 4$ disease was proven to be very helpful in documenting tumors invading adjacent contiguous structures, the aorta, and the tracheobronchial tree. Of similar value concerning possible airway invasion was bronchoscopy. Contraindications to resection included hepatic or other distant metastases, positive cytology for pleural effusion and/or peritoneal fluid, or airway invasion found by bronchoscopy.
Our data have been analyzed retrospectively. Follow-up was also obtained through personal interviews and examinations and telephone contacts with patients and families.

We have used the SPPS statistical package for the statistical analysis of the data. The survival analysis was performed using the Kaplan-Meier method for computing actuarial survival.

A $p$ value less than 0.05 was considered significant.

\section{Results}

Five hundred forty seven (73\%) of our patients were male and $203(27 \%)$ were female. The mean age was $62.04 \pm 12.25$ years.

Sixty patients $(8 \%)$ underwent esophageal replacement for benign disease, whereas 690 (92\%) for carcinoma. The age of the patient group with malignant disease ranged from 34 to 88 years (average 63 years).

Histopathologically, 545 (79\%) patients had adenocarcinoma and 145 (21\%) had squamous cell carcinoma.

The tumor was located in the upper third of the esophagus in 36 patients (5.2\%), the midthoracic esophagus in 51 (7.4\%), and lower third of the esophagus in 131 patients $(19 \%)$. In the majority of the cases (472 patients, $68.4 \%$ ), the carcinomas involved the gastroesophageal junction.

Postsurgical TNM staging [5] of the carcinomas based on histologic examination of the resected specimen is shown in Table 1.

Intraoperative complications

Seven hundred and fifty patients underwent esophagectomy without thoracotomy. There was no intraoperative death. The mean operative time was $137 \mathrm{~min}$, ranging from 120 to $180 \mathrm{~min}$. The average intraoperative blood loss was $315 \mathrm{~cm}^{3}$ (ranging from 300 to $350 \mathrm{~cm}^{3}$ ) and no blood transfusion was needed in $82 \%$ of cases (615 patients). There were three tracheal lacerations $(0.4 \%)$ that were managed intraoperatively, suturing the trachea via a sternotomy in one patient and via the cervical incision in

Table 1 Postsurgical TNM staging

\begin{tabular}{lcc}
\hline \multicolumn{2}{l}{ Postsurgical TNM staging } \\
\hline Stage & Number & Percentage \\
\hline O & 14 & 2.02 \\
I & 42 & 6.08 \\
IIA & 120 & 17.39 \\
IIB & 73 & 10.58 \\
III & 404 & 58.55 \\
IV & 37 & 5.35 \\
Total & 690 & 100 \\
\hline
\end{tabular}


the other two patients. Three patients $(0.4 \%)$ required splenectomy due to intraoperative injury. No instance of azygous vein injury was encountered.

Postoperative complications

Postoperative mediastinal bleeding was presented in six patients $(0.8 \%)$ requiring thoracotomy. There were also three cases of postoperative intraperitoneal hemorrhage needing a relaparotomy. Spleen injury was the source thereof in one patient and diffuse bleeding of the peritoneal cavity in the other two patients. In any case, no thoracotomy was required for controlling intraoperative bleeding.

Recurrent laryngeal nerve (RLN) injury occurred in six patients $(0.8 \%)$. This manifested through hoarseness due to vocal cord paresis and in all cases was transient resolving within 6 to 12 months.

There was no case of chylothorax. Pleural effusion developed in 170 patients $(22.7 \%)$. Spontaneous resolution occurred in 66 of them. In 57 patients, paracentesis was sufficient, whereas insertion of a chest tube was necessary in 47 of them. Clinically significant atelectasis or pneumonia was manifested in 36 patients $(4.8 \%)$. Thirty patients (4\%) presented abdominal wound infection, which was treated with drainage and antibiotics.

Anastomotic leak rate

We distinguish the anastomotic leak into silent radiologic leak, transient salivary fistula (stops within 5 days), and persistent salivary fistula (lasting more than 5 days). The incidence of anastomotic leak has been reduced over time, as shown in Table 2.

Interestingly, anastomotic leak rate was clearly higher when the substernal/subcutaneous route was used for esophageal replacement 30 leaks $(56 \%)$ in the 55 patients with substernal/subcutaneous placement of the graft.

Mortality rate

Postoperative mortality was defined as in-hospital death after surgery. The overall hospital mortality in our patients

Table 2 Anastomotic leak rate according to period

Anastomotic leak rate

\begin{tabular}{lllll}
\hline Period (years) & Patients $(n)$ & \multicolumn{4}{l}{ Leak rate $(\%)$} \\
\cline { 3 - 5 } & & Silent & Transient & Persistent \\
\hline $1981-1990$ & 255 & 11 & 9.4 & 20 \\
$1991-2000$ & 345 & 13.3 & 5.5 & 11.6 \\
$2001-2007$ & 150 & 16.6 & 4.76 & 6.35 \\
\hline
\end{tabular}

undergoing THE was $2.93 \%$ (22 patients). Mortality was mainly attributed to respiratory complications and sepsis (Table 3). There was no intraoperative death in our series of 750 patients.

\section{Length of hospital stay}

The average length of hospital stay was 11 days. One hundred and three patients $(13.7 \%)$ were ventilated following surgery, while 56 patients $(7.4 \%)$ required ventilation for more than $24 \mathrm{~h}$ due to respiratory failure.

Of the 728 patients discharged alive after THE, 145 patients $(19.33 \%)$ were discharged by the seventh postoperative day, 257 patients $(35.45 \%)$ between the seventh and tenth postoperative days, and 326 patients (45.25\%) later than the tenth postoperative day.

Out of 750 patients, 187 required admission in the intensive care unit (ICU). The length of stay in the ICU varied from 1 to 25 days (mean stay 9 days).

Functional results

Of the 728 patients discharged alive after THE, follow-up took place in 551, concerning functional results of esophageal substitute for up to 5 years (average 36 months). Three hundred seventy-four patients $(68 \%)$ had no dysphagia at all; 88 (16\%) experienced occasional dysphagia not requiring any treatment; $60(10.9 \%)$ experienced periodic dysphagia that needed occasional esophageal dilatations and $28(5.1 \%)$ experienced severe dysphagia that required repeated dilatations. No surgical intervention was required for correction of anastomotic stricture.

No regurgitation of gastric contents occurred in 351 patients $(63.8 \%)$, mild regurgitation when reclining or in the prone position after eating in 172 patients $(31.3 \%)$, and severe nocturnal regurgitation in 23 patients $(4.3 \%)$. Three patients $(0.6 \%)$ experienced respiratory complications due to aspiration.

The overall functional results of the patients followed up were characterized using the system of Orringer et al. [4, 6], as excellent (asymptomatic) in 258 patients (47\%), good (mild-no treatment) in 199 (36.2\%), fair (occasional treatment) in $64(11.7 \%)$, and poor (regular treatment) in $28(5.1 \%)$. These results are shown in Table 4.

Survival rate of patients with carcinoma

Of the 690 patients undergoing THE for carcinoma, 669 patients left hospital alive. Eighty two (12.2\%) of the above were lost to follow-up. The rest of the patients were followed up for a maximum of 72 months (mean follow-up 36 months). The Kaplan-Meier actuarial survival for the first 5 years after THE for carcinoma is shown in Fig. 1 and 
Table 3 Causes of hospital mortality among 750 patients

\begin{tabular}{ll}
\hline Cause of death & No. of deaths \\
\hline Malignant disease (690 patients) & $21(3 \%)$ \\
Respiratory insufficiency & 6 \\
Pneumonia & 5 \\
Myocardial infarction & 4 \\
Sudden death/cardiac arrest & 3 \\
Sepsis & 3 \\
Benign disease (60 patients) & $1(1.6 \%)$ \\
Myocardial infarction & 1 \\
Total & $22(2.9 \%)$ \\
\hline
\end{tabular}

Table 5. The overall 5-year survival rate was $21 \%$. The stage of the resected tumor was an important determinant of survival after THE.

Fifty-six patients (7.4\%) underwent neoadjuvant chemoradiotherapy at other centers before referral for surgery.

\section{Discussion}

Following a period in which THE was criticized [7-11], it has eventually been established as a standard method of esophageal resection for benign and malignant esophageal disease. Over a time of 25 years, we have performed THE in 750 patients. By focusing on this field of surgery, we have gained experience and consequently morbidity and mortality rates in our hands remain significantly low.

With respect to morbidity, our data will contain a thorough review of the complications in THE.

Recurrent laryngeal nerve injury, as manifested by early postoperative hoarseness, developed in ten patients $(1.33 \%)$. Hoarseness was usually transient and resolved in 1-3 months. To these patients with transient vocal cord paralysis, endoscopic evaluation of the larynx was carried out. Laryngoscopy was not performed routinely, only in cases where symptoms (hoarseness) developed. Possibly, some asymptomatic cases may have been missed. The reported incidence of this complication varies between 5\% and $20 \%[2,3,10,12-14]$. We believe that the low incidence of this injury, in our patients, is the result of the surgical technique we use for dissecting and mobilizing the cervical esophagus. Once the cervical incision along the medial border of the sternocleidomastoid muscle has been performed and the esophagus has been exposed, we grasp the cervical esophagus with a Babcock clamp and gently pull the esophagus away from the trachea. Then, we slowly encircle the esophageal wall with a dissector and proceed to gently dissect the cervical esophagus, while staying clear of the connective tissue where the RLN lies. With these maneuvers, we have reported a very low incidence of RLN injury.

In the first 96 cases of THE, we used to position a closed suction drain posterior to the cervical anastomosis, which was generally removed by the third postoperative day when the amount of drainage was tailing off.

However, 24 patients (25\%) presented with a neck abscess due to late anastomotic leak that occurred after the removal of the drain. These patients were treated by wound exposure and drainage. As a consequence, we altered our strategy regarding drain removal. Since then, we leave a closed suction drain in place for at least 10 days, so, even if an anastomotic leak occurs subsequently, the effluence drains away the anastomosis, which results in the development of a fistula but not an abscess. Since we have applied these measures, we have significantly eliminated the incidence of cervical wound abscesses, which overall accounts for 54 patients (9\%).

During the first years, pyloromyotomy was performed routinely in our institution, as it was generally believed that after truncal vagotomy and anatomical rearrangement a pyloric drainage procedure is necessary $[1,12,15,16]$.
Table 4 Functional results

Functional results after THE

\begin{tabular}{llr}
\hline Cervical dysphagia & No & $68 \%$ \\
& Mild with no treatment & $16 \%$ \\
& Periodic_occasional dilatation & $10.9 \%$ \\
Regurgitation & Severe & $5.1 \%$ \\
& No & $63.8 \%$ \\
Mild after eating & $31.3 \%$ \\
Overall functional results & Severe & $4.3 \%$ \\
& Severe with aspiration & $0.6 \%$ \\
& Excellent & $47 \%$ \\
& Good & $36.2 \%$ \\
& Fair & $11.7 \%$ \\
& Poor & $5.1 \%$ \\
\hline
\end{tabular}




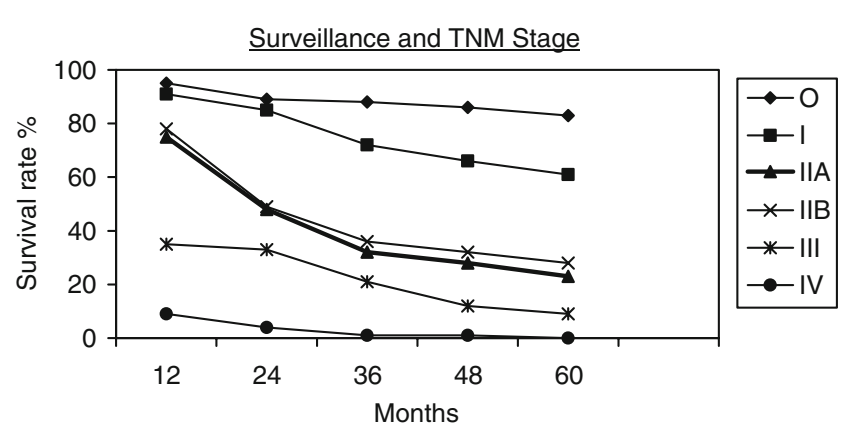

Fig. 1 Stage-dependent Kaplan-Meier actuarial survival curves

Complications such as dumping syndrome, diarrhea, or leakage from the pyloromyotomy were observed and attributed to pyloroplasty resulting in the above procedure being abandoned in all cases of THE [17-21].

Over a 25-year period, we have performed THE and reconstruction of the esophagus using the whole stomach or a gastric conduit, without pyloromyotomy or other drainage procedure, in more than 600 cases with good functional results in most cases. Overall, a pyloric dilatation was necessary in only one patient due to gastric stasis and vomiting (regurgitation), which led to significant weight gain. Furthermore, in a study of 65 of our patients with vagotomy, but without any pyloric drainage procedure, gastric emptying was evaluated scintigraphically [22].

Gastric emptying in the above patients was evaluated by having a radioisotope-labeled meal (eggs labeled with ${ }^{99} \mathrm{Tc}$ ) and by calculating half-time of emptying $\left(T_{1 / 2}\right)$. Their results were compared with those of healthy individuals and showed that only 15 patients $(23 \%)$ had prolonged $T_{1 / 2}$. From these 15 patients, only two (3\%) had postoperative symptoms of gastric stasis, with the first being cured with the use of prokinetic agents (domperidone) and only the second necessitating endoscopic balloon dilatation.

From the 50 patients (77\%) with normal $T_{1 / 2}$, six (9\%) of them showed symptoms of gastric stasis, but in any case the symptoms were not so disturbing as to require endoscopic treatment.

Consequently, we have concluded that pyloromyotomy is not only unnecessary but, on the contrary, may cause significant postoperative complications.

Multiple studies confirm our findings that a drainage procedure after truncal vagotomy in THE is not mandatory [17-21].

Some surgeons routinely place bilateral chest tubes during THE. At our institution, a chest tube is never inserted intraoperatively into any pleural cavity, even if entry into one or both pleural cavities is identified during surgery. Only prior to closing the abdominal wound do we ask the anesthesiologist to expand the lungs. Pleural effusion, in one or both pleural cavities, developed in $22.7 \%$ of our patients postoperatively, which was normally absorbed in 1 or 2 weeks. Of the above patients, $7.6 \%$ of them underwent thoracic paracentesis and only 6.26\% required the insertion of a chest tube postoperatively. Remarkably, chylothorax did not occur in any of our patients.

The usual practice regarding oral feeding of patients undergoing THE and cervical anastomosis was for patients to start on oral intake during the seventh postoperative day, providing there were no complications and the esophagogram with gastrografin did not show any leak $[1,12-16$, 23, 24]. During the first 10 years of esophagectomies, our group applied this strategy but found that sometimes, despite the fact that the esophagogram was negative for a leak, a salivary cervical fistula occurred on commencing on oral feeding. A normal esophagogram on day 7 does not exclude a subsequent development of a leak. After many modifications, finally, since a decade, we have applied the following tactic: the patient is discharged from the hospital by the seventh postoperative day unless postoperative complications necessitate a longer hospital stay. The patient is discharged home and receives enteral feeding via the feeding jejunostomy catheter, which remains in situ. On the 12th postoperative day, a cine-esophagogram is performed routinely, in an outpatient basis. If no radiologic leak is detected, enteral feeding is discontinued and clear fluids are administered progressively, gradually moving on to a normal diet within several days. If even a small silent radiologic leak is observed on a routine esophagogram, the patient continues on home enteral feeding for one more week at which time a new esophagogram takes place on an outpatient basis. We have observed that even a small radiologic leak can result in the development of mediastinitis, cervical abscesses, or fistula to the skin if the patient commences on oral feeding. During the first 10 years of THEs performed by our group with the "old" policy of only 1-week delay on commencing oral intake, the persistent fistulas' rate was as high as $20 \%$ (Table 2).Currently, the persistent fistula rate in our center is as low as $6.35 \%$, which is acceptable according to international literature $[12,14,16$, 18-20]. Furthermore, most of the presenting leaks and fistulas are of very low output and are treated conservatively.

Table 5 Survival rate after THE by tumor stage

\begin{tabular}{lll}
\hline TNM stage & 2-year survival (\%) & 5-year survival (\%) \\
\hline O & $89 \%$ & $83.3 \%$ \\
I & $85 \%$ & $61 \%$ \\
IIA & $48.7 \%$ & $23.3 \%$ \\
IIB & $49 \%$ & $28.1 \%$ \\
III & $33 \%$ & $9.1 \%$ \\
IV & $4 \%$ & $0 \%$ \\
\hline
\end{tabular}


The above modification in combination with the postponement of removing the cervical drain from day 3 to at least ten postoperative days has resulted in a significant reduction of the cervical fistulas' development.

\section{Conclusions}

THE is a demanding and highly specialized operation, suitable for the management of benign and malignant diseases of the esophagus, that should be performed in high-volume esophageal centers in order to minimize morbidity and mortality.

The changing trends and lessons learned in 25 years of cumulative experience in esophageal surgery have resulted to the formulation of some strategies, which although are not randomized, have limited, substantially, morbidity and mortality in our series:

1. The way we apply in approaching and mobilizing cervical esophagus has resulted in reduced incidence of recurrent laryngeal nerve injury (1.33\%).

2. Avoiding the performance of pyloromyotomy after truncal vagotomy protects from the occurrence of severe complications, such as dumping syndrome, diarrhea, or leakage from the myotomy.

3. Leaving in place a closed suction drain posteriorly to the cervical anastomosis for at least 10 days avoids the development of a troublesome cervical abscess.

4. Delaying oral feeding until at least postoperative day 12 results in elimination of persistent fistulas formation.

Open Access This article is distributed under the terms of the Creative Commons Attribution Noncommercial License which permits any noncommercial use, distribution, and reproduction in any medium, provided the original author(s) and source are credited.

\section{References}

1. Orringer MB, Sloan H (1978) Esophagectomy without thoracotomy. J Thorac Cardiovasc Surg 76:643

2. Katariya K, Harvey JC, Pina E, Beattie EJ (1994) Complications of transhiatal esophagectomy. J Surg Oncol 57:157. doi:10.1002/ jso. 2930570305

3. Gandhi SK, Naunheim KS (1997) Complications of transhiatal esophagectomy. Chest Surg Clin N Am 7:601

4. Orringer MB, Marshall B, Iannettoni MD (2001) Transhiatal esophagectomy for treatment of benign and malignant disease. World J Surg 25:196-203. doi:10.1007/s002680020019

5. Fleming ID, Cooper JS, Henson DE et al (eds) (1998) AJCC cancer staging handbook from the American Joint Committee on
Cancer Staging Manual, 5th edn. Lippincott-Raven, Philadelphia, 65-69

6. Orringer M, Marshall B, Chang A, Lee J, Pickens A, Lau C (2007) Two thousand transhiatal esophagectomies. Changing trends, lessons learned. Ann Surg 24630:363-374

7. Akiyama H, Tsutsuramu M, Udagawa H et al (1994) Radical lymph node dissection for cancer of the thoracic esophagus. Ann Surg 220:364-372. doi:10.1097/00000658-199409000-00012

8. Altorki NK, Girardi L, Skinner DB (1997) En block esophagectomy improves survival for stage III esophageal cancer. J Thorac Cardiovasc Surg 114:948-955. doi:10.1016/S0022-5223(97) 70009-6

9. Nishimaki T, Suzuki T, Kanda T et al (1999) Extended radical esophagectomy for superficially invasive carcinoma of the esophagus. Surgery 125:142-147

10. Swanson SJ, Batirel HF, Bueno R et al (2001) Transthoracic esophagectomy with radical mediastinal and abdominal lymph node dissection and cervical esophagogastrostomy for esophageal carcinoma. Ann Thorac Surg 72(6):1918-1924. doi:10.1016/ S0003-4975(01)03203-9

11. Johansson J, DeMeester TR, Hagen JA et al (2004) En block vs transhiatal esophagectomy for stage T3 N1 adenocarcinoma of the distal esophagus. Arch Surg 139(6):627-631. doi:10.1001/arch surg.139.6.627 discussion 631-3

12. Orringer MB, Marshall B, Iannettoni MD (1999) Transhiatal esophagectomy: clinical experience and refinements. Ann Surg 230(3):392-403. doi:10.1097/00000658-199909000-00012

13. Griffin S, Desai J, Charlton M et al (1989) Factors influencing mortality and morbidity following esophageal resection. Eur J Cardiothorac Surg 3:419-423. doi:10.1016/1010-7940(89)90051-1

14. Bousamra M, Haasler GB, Parviz M (2002) A decade of experience with transthoracic and transhiatal esophagectomy. Am J Surg 183:162-167. doi:10.1016/S0002-9610(01)00861-3

15. Linden PA, Sugarbaker DJ (2003) Techniques of esophageal resection. Semin Thorac Cardiovasc Surg 15(2):197-209. doi:10.1016/S1043-0679(03)70028-8

16. Lin J, Iannettoni MD (2005) Transhiatal esophagectomy. Surg Clin North Am 85:593-610. doi:10.1016/j.suc.2005.01.009

17. Angorn IB (1975) Oesophagogastrostomy without a drainage procedure in oesophageal carcinoma. Br J Surg 62:601-604. doi:10.1002/bjs. 1800620804

18. Hфlscher AH, Voit H, Siewert JR, Buttermann G (1988) Function of the intrathoracic stomach. In: Siewert JR, Hфlscher AH (eds) Diseases of the esophagus. Springer, Berlin, pp 660-663

19. Huang GJ, Zhang DC, Zhang DW (1985) A comparative study of resection of carcinoma of the esophagus with and without pyloroplasty. In: DeMeester TR, Skinner DB (eds) Esophageal disorders: pathophysiology and therapy. Raven, New York, pp 383-388

20. Chattopadhyay TK, Gupta S, Padhy AK, Kapoor VK (1991) Is pyloroplasty necessary following intrathoracic transposition of stomach? Results of a prospective clinical study. Aust N Z J Surg 61:366-369. doi:10.1111/j.1445-2197.1991.tb00236.x

21. Velanovich V (2003) Esophagogastrectomy without pyloroplasty. Dis Esophagus 16:243-245. doi:10.1046/j.1442-2050.2003.00337.x

22. Kounoudes $\mathrm{CH}$ Gastric emptying evaluation in patients with truncal vagotomy in esophagectomies without a drainage procedure. Ph.D. thesis, anecdotal

23. Hulscher JB, Jan Tijssen GP, Obertop H, Van Lanschot JJB (2001) Transthoracic versus transhiatal resection for carcinoma of the esophagus: a meta-analysis. Ann Thorac Surg 72:306-313. doi:10.1016/S0003-4975(00)02570-4

24. Atkins BZ, Shah AS, Hutcheson KA et al (2004) Reducing hospital morbidity and mortality following esophagectomy. Ann Thorac Surg 78:1170-1176. doi:10.1016/j.athoracsur.2004.02.034 